\title{
The impact of COVID-19 pandemic on patients with ANCA associated vasculitis
}

\author{
Sam Kant ${ }^{1} \cdot$ Adam Morris $^{2} \cdot$ Srekar Ravi $^{3} \cdot$ Lauren Floyd $^{2} \cdot$ Eric Gapud $^{4} \cdot$ Brendan Antichos $^{4} \cdot$ Ajay Dhaygude $^{2}$. \\ Phil Seo ${ }^{4}$ Duvuru Geetha ${ }^{1,4}$ (I)
}

Received: 11 August 2020 / Accepted: 27 September 2020 / Published online: 8 October 2020

(C) Italian Society of Nephrology 2020

\begin{abstract}
Introduction The coronavirus 2019 (COVID-19) pandemic has brought on challenges not only to acute care, but also chronic care of patients. Individuals maintained on immunosuppression appear to be especially susceptible to COVID-19 infection. Patients with ANCA-associated vasculitis (AAV) frequently require immunosuppression and may be at increased risk for developing COVID-19. The incidence and impact of COVID-19 on patients with AAV is currently not known. We aimed to investigate this impact via a telephone questionnaire-based patient survey and chart review.

Methods A cross-sectional study of AAV patients followed at two centers was conducted. Data regarding demographics, disease characteristics and therapy were confirmed by chart review. A telephone survey was conducted to ascertain symptoms and contact exposure related to COVID-19, as well as changes in health care delivery during the pandemic period between January and July, 2020.

Results Of the 206 patients surveyed, the median age was 64 years, $51 \%$ were female and mean (SD) disease duration was 7 (5) years. The majority had kidney $(n=160)$ and lung $(n=108)$ involvement. Seventy-five percent $(n=155)$ were receiving immunosuppression, with 77 patients $(50 \%)$ receiving rituximab during the pandemic period. Of the 10 patients tested for severe acute respiratory syndrome coronavirus 2 (SARS CoV-2) by PCR, three were positive. Patients had a significant disruption in care; none had an in-person visit and $69 \%$ had a telemedicine consultation. Rituximab maintenance was postponed in 21 patients. Twelve patients experienced disease relapse.

Conclusion The incidence of COVID-19 in patients with AAV appears to be similar to that of the general population. For a patient population that requires active clinical surveillance, there is significant disruption in care as a result of the pandemic. Reduction of immunosuppression may not be indicated, and the risk of relapse likely far outweighs the risk of COVID-19.
\end{abstract}

Electronic supplementary material The online version of this article (https://doi.org/10.1007/s40620-020-00881-3) contains supplementary material, which is available to authorized users.

Duvuru Geetha

gduvura@jhmi.edu

1 Division of Nephrology, Department of Medicine, Johns Hopkins University School of Medicine, Baltimore, MD, USA

2 Royal Preston Hospital, Lancashire, UK

3 Florida Atlantic University, Boca Raton, FL, USA

4 Division of Rheumatology, Department of Medicine, Johns Hopkins University School of Medicine, Baltimore, MD, USA

\section{Introduction}

As the severe acute respiratory syndrome coronavirus 2 (SARS-CoV-2) disease 2019 (COVID-19) pandemic has evolved, there is concern that immunosuppressed patients, including those with ANCA-associated vasculitis (AAV), may be at increased risk for infection with COVID-19. Initial data involving patients with chronic arthritis on biologic and synthetic disease-modifying anti-rheumatic agents (DMARDs) did not demonstrate an association between immunosuppressive drugs and an increased risk of respiratory complications from COVID-19 [1]. However, a larger analysis of 600 patients with rheumatic disease demonstrated that patients on prednisone $>10 \mathrm{mg} /$ day are at higher risk of hospitalization [2]. The incidence and impact of COVID-19 on care of patients with AAV is currently not known. We 
aimed to investigate this impact via a COVID-19-specific telephone survey of AAV patients followed at two centers.

\section{Methods}

A cross-sectional study was conducted at the Johns Hopkins Hospital Vasculitis Center (Baltimore, Maryland, USA) and Royal Preston Hospital (Lancashire, UK). A COVID19-specific telephone survey of all consecutive AAV patients followed at both centers was conducted (Supplement S1). Data regarding demographics, disease characteristics, comorbidities and therapy were confirmed by review of the electronic medical record. Information regarding current and previous therapies was collected. Ethical approval for this study was received from the institutional review board at Johns Hopkins University School of Medicine and the UK Health Research Authority and Confidentiality Advisory Group. All except one rituximab infusion at Preston was delivered in a COVID-free, non-acute clinical area.

We contacted patients between May 1st and July 23rd, 2020 to ascertain the presence of symptoms and contact exposure related to COVID-19, along with results of nasopharyngeal swabs. We also sought information regarding practice of personal protective measures (PPMs), contact exposure, travel to high-risk areas in Europe, United States and China, quarantine status and effect on delivery of health care during the period of pandemic from January to June 2020.

Descriptive data are presented as mean with standard deviation and median with range. Characteristics between the two cohorts are compared using the 2 tailed T-test for continuous variable and chi-square test or Fisher's exact test for categorical variables. All tests of significance were two sided and differences were considered significant if the p-value was less than 0.05 .

\section{Results}

Of 206 patients, the median age was 64 years, $51 \%$ were female and the mean (SD) disease duration was 7 (5) years. The majority had kidney $(n=160,78 \%)$ and lung $(n=108$, $52 \%)$ involvement, with $36 \%(\mathrm{n}=75)$ having sino-nasal disease. Significant differences in age $(\mathrm{p}=0.003)$, race $(p=0.002)$, disease type $(p=0.3)$, and co-morbidities $(p=0.001)$ were identified between the two cohorts. A history of relapsing disease was present in $39 \%(\mathrm{n}=81)$, with $64 \%(\mathrm{n}=132)$ and $77 \%(\mathrm{n}=158)$ having prior exposure to cyclophosphamide (CYC) and rituximab, respectively. Seventy-five percent $(n=155)$ were receiving immunosuppression, with $50 \%(\mathrm{n}=77)$ of these patients receiving rituximab during the pandemic period. Details of regimens of immunosuppression and co-morbidities are presented in Tables 1 and 2 .

Since the start of the pandemic (January 2020), all but one patient followed PPMs to decrease the risk of COVID19 infection. Nearly all patients reported using a facemask/ shield (97\%), practiced social distancing (98\%), washing hands frequently (92\%) and 177 patients (83\%) reported following all three PPMs. A majority $(n=193)$ of patients who completed the survey did not have symptoms of infection. Seven patients experienced cough, 6 had dyspnea, 4 patients each developed fever and mylagias. Seven of the symptomatic patients were not tested for COVID-19 infection and reported self-resolution of symptoms at the time of the survey. In total ten (four asymptomatic and six symptomatic) patients underwent PCR testing for COVID-19, of which only 3 were positive. Most patients self-quarantined after potential/confirmed COVID-19 exposure, with none developing COVID-19. Regarding delivery of care, $69 \%$ had video visits, none had in-person visits, and $13 \%$ of visits were rescheduled. Rituximab maintenance therapy was postponed in 21 patients. Twelve patients experienced disease relapse during this period. COVID-19 specific variables are represented in Table 3.

Of the three patients who developed COVID-19, two were PR3 positive with one MPO positive. Two patients were on remission maintenance with azathioprine, while one was receiving remission induction with rituximab for active vasculitis. All patients had symptoms of fever and dyspnea, with a duration of 1-9 days prior to confirmation of COVID-19. The two patients in remission required admission to intensive care; one required supplemental oxygen and the other non-invasive ventilation. In both cases, maintenance immunosuppression was held, and one received steroids. The third patient received a reduced dose of $1 \mathrm{~g}$ rituximab for remission induction as they tested positive for COVID-19 within $24 \mathrm{~h}$ of drug administration. They did not experience any serious complications of infection and were monitored as an outpatient. Recovery from COVID-19 identified by symptom resolution in three patients (negative viral testing in one patient). Median time to recovery was 25 days (range 3-64 days).

Twelve patients had disease relapse. Details of disease characteristics, maintenance therapy and immunosuppression at the time of relapse are presented in Table 4. Mean (SD) age was 67 (10) years with a mean (SD) disease duration of 5(3) years. Seven were PR3-ANCA positive and two had a history of relapsing disease. Organs involved at relapse included kidney in four patients and lung in three patients, while there was ENT involvement in three patients and neuropathy in two. At the time of relapse, all patients were on maintenance immunosuppression. Among the five patients on rituximab for remission maintenance, one missed a scheduled rituximab infusion, and rituximab infusion was 
Table 1 Baseline demographics and clinical characteristics

\begin{tabular}{|c|c|c|c|c|c|}
\hline AAV-specific variables & $\begin{array}{l}\text { Number of subjects } \\
n=206\end{array}$ & $\begin{array}{l}\text { Entire cohort } \\
\mathrm{n}=206\end{array}$ & $\begin{array}{l}\text { Hopkins } \\
\mathrm{n}=117\end{array}$ & $\begin{array}{l}\text { Preston } \\
\mathrm{n}=89\end{array}$ & $\mathrm{p}$-value \\
\hline Age & Mean years (SD) & $64(14)$ & $61(15)$ & $68(11)$ & 0.0003 \\
\hline Gender, n (\%) & Female & $106(51)$ & $66(56)$ & $40(45)$ & 0.1 \\
\hline \multirow[t]{3}{*}{ Race, $\mathrm{n}(\%)$} & White & $184(89)$ & $97(83)$ & 87 (98) & \\
\hline & African-American & $12(6)$ & $12(10$ & $0(0)$ & 0.002 \\
\hline & Other & $10(5)$ & $8(7)$ & $2(2)$ & \\
\hline \multirow[t]{3}{*}{ Diagnosis, n (\%) } & GPA & $116(56)$ & $63(54)$ & $53(59)$ & \\
\hline & MPA & $68(33)$ & $46(39)$ & $22(25)$ & 0.03 \\
\hline & EGPA & $22(11)$ & $8(7)$ & $14(16)$ & \\
\hline Disease duration & Mean years (SD) & $6.77(5)$ & $7.2(6)$ & $6.2(4)$ & 0.2 \\
\hline \multirow[t]{3}{*}{ ANCA type, n (\%) } & PR3 & $104(50)$ & $59(50)$ & $45(51)$ & 0.7 \\
\hline & MPO & $93(45)$ & $55(47)$ & $38(43)$ & \\
\hline & Negative & $9(4)$ & $3(3)$ & $6(7)$ & \\
\hline \multirow[t]{10}{*}{ Organs involved, n (\%) } & Kidney & $161(78)$ & $97(83)$ & $64(72)$ & 0.06 \\
\hline & Lung & $108(52)$ & $68(58)$ & $40(45)$ & 0.06 \\
\hline & Ears & $16(8)$ & $12(10)$ & $4(4)$ & 0.1 \\
\hline & Eyes & $29(14)$ & $19(16)$ & $10(11)$ & 0.3 \\
\hline & Nose and sinus & $75(36)$ & $42(36)$ & $33(37)$ & 0.9 \\
\hline & Heart & $3(1)$ & $2(2)$ & $1(1)$ & 0.7 \\
\hline & GI tract & $7(3)$ & $5(4)$ & $2(2)$ & 0.4 \\
\hline & Nerves & $29(14)$ & $16(14)$ & $13(15)$ & 0.8 \\
\hline & Skin & $32(16)$ & $14(12)$ & $18(20)$ & 0.1 \\
\hline & Joints & $35(17)$ & $21(18)$ & $14(16)$ & 0.7 \\
\hline History of vasculitis relapse, $\mathrm{n}(\%)$ & & $81(39)$ & $42(36)$ & $39(44)$ & 0.3 \\
\hline Current use of ACE inhibitors, $n(\%)$ & & $40(19)$ & $29(25)$ & $11(12)$ & 0.1 \\
\hline Current use of ARBs, n (\%) & & $45(22)$ & $25(21)$ & $20(22)$ & 0.1 \\
\hline \multirow[t]{10}{*}{ Comorbidities, n (\%) } & & & & & 0.001 \\
\hline & Hypertension & $129(63)$ & $91(78)$ & $38(43)$ & $2 \mathrm{E}-7$ \\
\hline & Diabetes & $24(12)$ & $12(10)$ & $12(13)$ & 0.5 \\
\hline & Heart disease & $23(11)$ & $11(9)$ & $12(13)$ & 0.4 \\
\hline & Cerebrovascular disease & $4(2)$ & $1(1)$ & $3(3)$ & 0.2 \\
\hline & Chronic kidney disease & $128(62)$ & $68(58)$ & $62(70)$ & 0.09 \\
\hline & Dialysis & $6(3)$ & $5(4)$ & $1(1)$ & 0.2 \\
\hline & Organ transplant & $8(4)$ & $8(7)$ & $0(0)$ & 0.01 \\
\hline & Cancer & $15(7)$ & $5(4)$ & $10(11)$ & 0.06 \\
\hline & No comorbidities & $26(13)$ & $17(14)$ & $9(10)$ & 0.3 \\
\hline
\end{tabular}

Table 2 Details of immunosuppression regimen

\begin{tabular}{|c|c|c|c|c|c|c|}
\hline & \multicolumn{3}{|l|}{ Prior use } & \multicolumn{3}{|l|}{ Current use } \\
\hline & Entire cohort & Hopkins & Preston & Entire cohort & Hopkins & Preston \\
\hline Cyclophosphamide, n (\%) & $132(64)$ & $61(52)$ & $71(80)$ & $6(3)$ & $0(0)$ & $6(7)$ \\
\hline Rituximab, n (\%) & $158(77)$ & $95(81)$ & $63(71)$ & $107(52)$ & $54(46)$ & $53(59)$ \\
\hline Received rituximab after June 2019, n (\%) & - & & & $105(51)$ & $58(50)$ & $47(53)$ \\
\hline Rituximab use during pandemic period, n (\%) & - & & & $77(38)$ & $39(33)$ & $38(43)$ \\
\hline Prednisone, $\mathrm{n}(\%)$ & 204 (99) & $116(99)$ & $88(99)$ & $96(47)$ & $53(45)$ & $43(48)$ \\
\hline Methotrexate, n (\%) & $21(10)$ & $12(10)$ & $9(10)$ & $6(3)$ & $4(3)$ & $2(2)$ \\
\hline Azathioprine, n (\%) & $63(31)$ & $33(28)$ & $30(34)$ & $17(8)$ & $9(8)$ & $8(9)$ \\
\hline Mycophenolate mofetil, n (\%) & $41(20)$ & $16(14)$ & $25(28)$ & $22(11)$ & $11(9)$ & $11(12)$ \\
\hline Leflunomide, n (\%) & $3(1)$ & $3(3)$ & $0(0)$ & $3(1)$ & $3(3)$ & $0(0)$ \\
\hline
\end{tabular}


Table 3 COVID-19 exposure, health care delivery and outcomes

\begin{tabular}{|c|c|}
\hline Contact with COVID-19 infected individual, n (\%) & $5(2)$ \\
\hline Traveled to high risk areas since December 2019, n (\%) & $3(1)$ \\
\hline Quarantined for COVID-19 infection, n (\%) & $3(1)$ \\
\hline \multicolumn{2}{|l|}{ Symptoms, n (\%) } \\
\hline No symptoms & $193(94)$ \\
\hline Fever & $4(2)$ \\
\hline Cough & $7(3)$ \\
\hline Dyspnea & $6(3)$ \\
\hline Headache & $2(1)$ \\
\hline Altered smell/taste & $1(0)$ \\
\hline Sore throat & $2(1)$ \\
\hline Muscle ache & $4(2)$ \\
\hline Nausea, vomiting, diarrhea & $2(1)$ \\
\hline Tested for C0VID-19, n (\%) & $10(5)$ \\
\hline \multicolumn{2}{|l|}{ COVID-19 test result, $\mathrm{n}(\%)$} \\
\hline Positive & $3(30)$ \\
\hline Negative & $7(70)$ \\
\hline \multicolumn{2}{|l|}{ Relapse during pandemic, n (\%) } \\
\hline Yes & $12(6)$ \\
\hline \multicolumn{2}{|l|}{ Measures taken to prevent COVID-19, $\mathrm{n}(\%)$} \\
\hline None & $1(0)$ \\
\hline Social distancing & $202(98)$ \\
\hline Face mask & $199(97)$ \\
\hline Hand washing & $190(92)$ \\
\hline All of the above & $171(83)$ \\
\hline \multicolumn{2}{|l|}{ Changes in health care during pandemic period, $\mathrm{n}(\%)$} \\
\hline None & $23(11)$ \\
\hline Regular in-person visit with physician & $0(0)$ \\
\hline Video visits with physician & $142(69)$ \\
\hline Rescheduled clinic visits & $26(13)$ \\
\hline Missed medications for at least 1 day & $1(0)$ \\
\hline Reduced medication dosage & $14(7)$ \\
\hline Maintenance rituximab infusion postponed & $21(10)$ \\
\hline Decreased blood draw frequency & $33(16)$ \\
\hline
\end{tabular}

postponed in a second patient in the setting of the pandemic. Rituximab with prednisone was used for remission induction treatment for these relapses in nine patients and one patient each received induction therapy with cyclophosphamide and prednisone, increase in doses of azathioprine, trimethoprim-sulfamethoxazole and prednisone. There were no cases of COVID-19 infection among patients experiencing disease relapse.

\section{Discussion}

The era of the COVID-19 pandemic has been challenging for longitudinal management of patients with chronic disease. Health authorities continue to advise that patients on immunosuppression are at higher risk for COVID-19 infection [3]. There is no current evidence-based guidance on the use of immunosuppression in patients with AAV during the pandemic, a disease frequently associated with high rates of relapse in the absence of maintenance therapy. Additionally, patients with rheumatic diseases have not been included in many large studies of patients with COVID-19 [4-6]. This hinders understanding of susceptibility for infection, along with outcomes associated with COVID-19 in this population.

Preliminary data regarding the incidence of COVID-19 in patients with autoimmune diseases are available via a survey done in Tuscany, Italy [7]. An incidence rate of $0.22 \%$ has been reported, comparable to that observed in the general population of Tuscany. However, $<10 \%(n=40)$ of patients in this cohort represented patients with AAV, with none reported to have COVID-19. A Spanish consortium reported that COVID-19 disease course and mortality in patients with rheumatic disease on biologic therapies did not differ from the general population [8]. Further, the incidence of hospitalizations among patients with autoimmune diseases is similar to patients with COVID-19 in the general population [9]. Additionally, all of the aforementioned report incidence and clinical course of COVID-19 in patients with rheumatic disease at large, with the inclusion of only a minority of patients with AAV (Table S1). With respect to our study, the overall incidence of COVID-19 in our cohort of AAV patients is $1.4 \%$, which is close to the current cumulative incidence of $1.2 \%$ in the United States [10]. These findings are encouraging and demonstrate that patients with AAV on immunosuppression may not be at higher risk for COVID19 in comparison to the general population. The majority of patients surveyed followed guidelines with personal protective measures, which mitigates infection in this high-risk cohort.

Regular outpatient surveillance with serial measurement of ANCA titers and B-cell kinetics are the best practice for detection of disease relapse $[11,12]$. Measures to decrease healthcare encounters were evident in most patients. Various centers will need to devise alternate methods to adapt to conditions borne as a result of the pandemic, balancing potential viral exposures with the need for robust monitoring of AAV.

Based on experience from SARS-CoV and MERS-CoV, glucocorticoid use has been associated with delayed viral clearance from blood and the respiratory tract $[13,14]$. Minimizing the use of glucocorticoids may be another approach to lessen the effects of COVID-19 in patients with AAV. All patients receiving maintenance steroid therapy from both centers were on less than $10 \mathrm{mg} /$ day of prednisone (most on $<5 \mathrm{mg}$ /day), with none requiring hospitalization. This further supports data from the COVID-19 Global Rheumatology alliance which showed that steroid dosage $>10 \mathrm{mg}$ is associated with higher rates of hospitalization [2]. 
Table 4 Characteristics and outcome of relapses during pandemic

\begin{tabular}{|c|c|c|c|c|c|c|c|}
\hline Patient no & $\begin{array}{l}\text { Disease } \\
\text { duration } \\
\text { (years) }\end{array}$ & Disease type & ANCA type & Prior maintenance $\mathrm{Tx}$ & IS at time of relapse & $\begin{array}{l}\text { Organs involved at } \\
\text { relapse }\end{array}$ & Relapse Tx \\
\hline 1 & 3 & MPA & MPO & RTX & $\mathrm{RTX}^{\mathrm{a}}$ & Kidney & $\mathrm{RTX}+\mathrm{P}$ \\
\hline 2 & 4 & GPA & PR3 & RTX & $\mathrm{RTX}^{\mathrm{b}}$ & Kidney, lung & $\mathrm{RTX}+\mathrm{P}$ \\
\hline 3 & 10 & MPA & MPO & $\mathrm{MMF}+\mathrm{P}$ & $\mathrm{MMF}+\mathrm{P}$ & Kidney & RTX \\
\hline 4 & 1.9 & MPA & MPO & $\mathrm{RTX}+\mathrm{P}$ & $\mathrm{RTX}+\mathrm{P}$ & Peripheral nerves & $\mathrm{RTX}+\mathrm{P}$ \\
\hline 5 & 2 & MPA & MPO & None & $\mathrm{MMF}+\mathrm{P}$ & Lung & RTX \\
\hline 6 & 4.6 & GPA & PR3 & $\mathrm{MMF}+\mathrm{P}$ & $\mathrm{MMF}+\mathrm{P}$ & ENT & TMP-SMZ \\
\hline 7 & 2 & GPA & PR3 & $\mathrm{P}+\mathrm{TMP}-\mathrm{SMZ}$ & $\mathrm{P}+\mathrm{TMP}-\mathrm{SMZ}$ & Lung & RTX \\
\hline 8 & 8 & GPA & PR3 & $\mathrm{AZA}+\mathrm{TMP}-\mathrm{SMZ}$ & AZA & ENT & $\mathrm{AZA}+\mathrm{TMP}-\mathrm{SMZ}$ \\
\hline 9 & 2 & EGPA & PR3 & RTX + TMP-SMZ & RTX & Kidney & $\mathrm{RTX}+\mathrm{P}$ \\
\hline 10 & 2 & GPA & PR3 & $\mathrm{MTX}+\mathrm{P}$ & $\mathrm{MTX}+\mathrm{P}$ & Eyes & $\mathrm{RTX}+\mathrm{P}$ \\
\hline 11 & 6 & GPA & Negative & $\mathrm{MMF}+\mathrm{P}$ & $\mathrm{MMF}+\mathrm{P}$ & ENT & $\mathrm{CYC}+\mathrm{P}$ \\
\hline 12 & 10.5 & GPA & PR3 & RTX & RTX & Peripheral nerves & $\mathrm{RTX}+\mathrm{P}$ \\
\hline
\end{tabular}

$T x$ treatment, $M P A$ microscopic polyangiitis, GPA granulomatosis with polyangiitis, EGPA eosinophilic granulomatosis with polyangiitis, $M P O$ myeloperoxidase, $P R 3$ proteinase 3, $R T X$ rituximab, $M M F$ mycophenolate mofetil, $P$ prednisone, $T M P-S M Z$ trimethoprim-sulfamethoxazole, $A Z$ : azathioprine, $M T X$ methotrexate, $C Y C$ cyclophosphamide

${ }^{a}$ RTX postponed by physician

${ }^{\mathrm{b}} \mathrm{RTX}$ infusion canceled by patient

There continues to be a lack of evidence-based guidance for the use of rituximab in patients with AAV during the pandemic. Experience with rituximab use in the context of COVID-19 has been reported in multiple sclerosis and pemphigus vulgaris $[15,16]$. Patients with multiple sclerosis on anti-CD20 therapy had adequate resolution of COVID-19 [15]. A case series of pemphigus vulgaris patients on rituximab demonstrated that only $5 / 147$ patients developed COVID-19, with none requiring hospitalization [16]. An Italian survey of 159 pediatric patients with relapsing nephrotic syndrome treated with anti-CD20 antibodies identified no cases of COVID-19 infection [17]. Severe respiratory presentations of COVID-19 have been reported immediately post rituximab therapy in patients with rheumatic disease [18, 19], albeit one of the reports does hypothesize that rituximab may have limited the cytokine storm and delayed worsening of clinical status of a patient with AAV [19]. Indeed, our patient tested positive for COVID-19 immediately post rituximab administration and had an uneventful course despite being elderly. In addition, a case series of patients with autoimmune disease has shown that baseline biologic therapy use is not associated with worse COVID-19 outcomes [9]. Twenty-one (30\%) of 77 patients on maintenance rituximab therapy had postponement of infusion in our study. Two (10\%) of the 21 patients with postponement had evidence of disease relapse. Most concerningly, $6 \%(n=12)$ of patients in the overall cohort had disease relapse. Furthermore, all relapsing patients were on maintenance immunosuppression with no alteration to their regimen in all but two patients.
These high relapse rates may be attributable to lack of inperson consultation required to gauge extra-renal relapses. Based on these data, it is possible that the risk of relapse may outweigh the risk of COVID-19 infection in the setting of immunosuppression withdrawal.

Our study is not without limitations. The study was conducted in centers with different healthcare systems, with differences in incidence of COVID-19 and the majority of patients being Caucasian and an absence of a control group for comparison. Only ten patients underwent COVID-19 testing and hence the incidence of asymptomatic carrier state or masked symptoms related to COVID-19 cannot be ascertained, especially whilst being on immunosuppression. The lack of a control group limits epidemiological conclusions from this study. Despite these limitations, this is the first study assessing the impact of COVID-19 infection in AAV patients, and the majority of the patients surveyed were on immunosuppression, representative of the AAV population.

Our data demonstrate that continual monitoring of AAV patients during the pandemic is paramount. Patients with AAV have similar incidence of COVID-19 in comparison to the general population. These data also support that withdrawal of maintenance therapy to prevent COVID-19 may not be advisable in most circumstances and may be associated with disease relapse. A balanced approach to continuation versus withdrawal of therapy needs to be tailored to the risk of infection and disease relapse in the era of the pandemic. Continued safe use of immunosuppression is on the basis that all patients utilize PPMs as demonstrated in our cohort. Our experience with relapse treatment demonstrates 
that current induction therapy can be safely administered during the pandemic.

\section{Compliance with ethical standards}

Conflict of interest DG reports being consultant to ChemoCentryx and Aurinia. The other authors do not report any conflict of interest.

Ethical statement The study was approved by the Institutional review board of both the hospitals.

\section{References}

1. Monti S, Balduzzi S, Delvino P, Bellis E, Quadrelli VS, Montecucco C (2020) Clinical course of COVID-19 in a series of patients with chronic arthritis treated with immunosuppressive targeted therapies. Ann Rheum Dis 79(5):667-668. https://doi. org/10.1136/annrheumdis-2020-217424

2. Gianfrancesco M, Hyrich KL, Al-Adely S et al (2020) Characteristics associated with hospitalisation for COVID-19 in people with rheumatic disease: data from the COVID-19 Global Rheumatology Alliance physician-reported registry. Ann Rheum Dis. https ://doi.org/10.1136/annrheumdis-2020-217871

3. CDC (2020) People of any age with underlying medical conditions. https://www.cdc.gov/coronavirus/2019-ncov/need-extra -precautions/people-with-medical-conditions.html?CDC_AA refVal=https $\% 3 \mathrm{~A} \% 2 \mathrm{~F} \% 2 \mathrm{Fwww} . c d c$.gov $\% 2 \mathrm{Fcoronavirus} \% 2 \mathrm{~F} 201$ 9-ncov\%2Fneed-extra-precautions $\% 2$ Fgroups-at-higher-risk. html\#immunocompromised-state. Accessed 15 July 2020

4. Guan W, Ni Z, Hu Y et al (2020) Clinical characteristics of coronavirus disease 2019 in China. N Engl J Med 382(18):1708-1720. https://doi.org/10.1056/NEJMoa2002032

5. Richardson S, Hirsch JS, Narasimhan M et al (2020) Presenting characteristics, comorbidities, and outcomes among 5700 patients hospitalized with COVID-19 in the New York City Area. JAMA. https://doi.org/10.1001/jama.2020.6775

6. Grasselli G, Zangrillo A, Zanella A et al (2020) Baseline characteristics and outcomes of 1591 patients infected with SARSCoV-2 admitted to ICUs of the Lombardy Region, Italy. J Am Med Assoc 323(16):1574-1581. https://doi.org/10.1001/ jama.2020.5394

7. Emmi G, Bettiol A, Mattioli I et al (2020) SARS-CoV-2 infection among patients with systemic autoimmune diseases. Autoimmun Rev 19(7):102575. https://doi.org/10.1016/j.autrev.2020.102575

8. Sanchez-Piedra C, Diaz-Torne C, Manero J et al (2020) Clinical features and outcomes of COVID-19 in patients with rheumatic diseases treated with biological and synthetic targeted therapies.
Ann Rheum Dis 79(7):988-990. https://doi.org/10.1136/annrh eumdis-2020-217948

9. Chen A, Hudesman D, Adhikari S et al (2020) Covid-19 in immune-mediated inflammatory diseases-case series from New York. N Engl J Med 383(1):85-88. https://doi.org/10.1056/ NEJMc2009567

10. CDC (2020) CoVID-19 surveillance. https://www.cdc.gov/coron avirus/2019-ncov/cases-updates/cases-in-us.html. Accessed 31 July 2020

11. Nataraja A, Mukhtyar C, Hellmich B, Langford C, Luqmani R (2007) Outpatient assessment of systemic vasculitis. Best Pract Res Clin Rheumatol 21(4):713-732. https://doi.org/10.1016/j. berh.2007.01.004

12. Boomsma MM, Stegeman CA, van der Leij MJ et al (2000) Prediction of relapses in Wegener's granulomatosis by measurement of antineutrophil cytoplasmic antibody levels-a prospective study. Arthritis Rheum 43(9):2025-2033

13. Lee N, Allen Chan KC, Hui DS et al (2004) Effects of early corticosteroid treatment on plasma SARS-associated coronavirus RNA concentrations in adult patients. J Clin Virol 31(4):304-309. https ://doi.org/10.1016/j.jcv.2004.07.006

14. Russell CD, Millar JE, Baillie JK (2020) Clinical evidence does not support corticosteroid treatment for 2019-nCoV lung injury. Lancet 395(10223):473-475. https://doi.org/10.1016/S0140 $-6736(20) 30317-2$

15. Meca-Lallana V, Aguirre C, Río B, Cardeñoso L, Alarcon T, Vivancos J (2020) COVID-19 in 7 multiple sclerosis patients in treatment with ANTI-CD20 therapies. Mult Scler Relat Disord 44:102306. https://doi.org/10.1016/j.msard.2020.102306

16. Shahidi-Dadras M, Abdollahimajd F, Ohadi L et al (2020) COVID-19 in Pemphigus vulgaris patients with previous rituximab therapy: a tele-medicine experience. J Dermatol Treat. https ://doi.org/10.1080/09546634.2020.1789041

17. Angeletti A, Drovandi S, Sanguineri F et al (2020) COVID-19 in children with nephrotic syndrome on anti-CD20 chronic immunosuppression. Clin J Am Soc Nephrol. https://doi.org/10.2215/ CJN.06400420

18. Schulze-Koops H, Krueger K, Vallbracht I, Hasseli R, Skapenko A (2020) Increased risk for severe COVID-19 in patients with inflammatory rheumatic diseases treated with rituximab. Ann Rheum Dis. https://doi.org/10.1136/annrheumdis-2020-218075

19. Guilpain P, Le Bihan C, Foulongne V et al (2020) Rituximab for granulomatosis with polyangiitis in the pandemic of covid-19: lessons from a case with severe pneumonia. Ann Rheum Dis. https ://doi.org/10.1136/annrheumdis-2020-217549

Publisher's Note Springer Nature remains neutral with regard to jurisdictional claims in published maps and institutional affiliations. 